Génét. Sél. Evol., 1988, 20 (3), 321-342

\title{
Comparaison des races bovines Charolaise, Limousine et Maine-Anjou en race pure et en intercroisement. 1. Objectif et dispositif expérimental
}

\author{
F. MÉNISSIER *, B. BIBÉ **, B. PERREAU ${ }^{* * *}$ et B. VISSAC **** \\ Institut National de la Recherche Agronomique \\ * - Station de Génétique Quantitative et Appliquée, \\ Centre de Recherches de Jouy-en-Josas, 78350 Jouy-en-Josas, France \\ ** - Station d'Amélioration Génétique des Animaux, \\ Centre de Recherches de Toulouse, B.P. 27, 31326 Castanet-Tolosan, France \\ *** - Station de Génétique Quantitative et Appliquée, \\ Domaine expérimental de Galle, 18520 Avord, France \\ **** - Département de Recherches sur les Systèmes Agraires et le Développement, \\ 147, rue de l'Université, 75341 Paris Cedex 07, France
}

\begin{abstract}
Résumé
Les races à viande françaises ont été très largement utilisées pour améliorer la production de viande bovine durant les années 60 . Les connaissances acquises sur ces races restaient insuffisantes pour optimiser leur utilisation. Une expérience d'évaluation des races Charolaise, Limousine et Maine-Anjou en race pure et en croisement entre elles a été mise en œuvre de 1970 à 1985 pour comparer leurs effets génétiques sur les aptitudes bouchères et sur les qualités maternelles. Le contexte de l'expérience, le choix du matériel animal, le dispositif expérimental et l'organisation de la conduite et des contrôles sont présentés. Le matériel animal fondateur a été constitué par un échantillon de génisses de race pure (66 à 71 animaux par race) achetées au sevrage dans des élevages de production et par la semence de taureaux de race pure (six par race) choisis parmi ceux utilisés pour l'insémination artificielle en France. Des génisses et de la semence de taureaux Hereford ont été ajoutées pour servir de connexion avec les essais étrangers. Le dispositif expérimental a été basé sur trois phases d'accouplements selon les générations : plan de croisement diallèle (I), croisements de femelles de race pure et croisées F1 avec des mâles de race pure (II), croisements de femelles croisées $\mathrm{F} 1$ et trois races avec des mâles de diverses races (III). Ce dispositif a permis, outre les comparaisons des types génétiques, l'estimation des effets additifs et d'hétérosis directs et maternels $\left(G^{I}, G^{M}, H^{I}, H^{M}\right)$. La conduite et les contrôles des bovins ont été réalisés dans plusieurs implantations expérimentales. L'originalité des résultats attendus demeure malgré la durée de l'expérience.
\end{abstract}

Mots clés: bovins à viande, races françaises, dispositif expérimental, effets génétiques, qualités bouchères, qualités maternelles. 


\section{Summary \\ Comparison between Charolais, Limousin and Maine-Anjou breeds in pure- and crossbreeding \\ 1. Goal and experimental design}

French beef breeds were extensively used to improve beef production during the sixties. However, the knowledge gathered about these breeds was insufficient for their optimum use. From 1970 to 1985, an evaluation experiment was set up in pure- and crossbred Charolais, Limousin and Maine-Anjou breeds for comparing their genetic effects on lean production capacity and maternal abilities. The experimental conditions, choice of animals, experimental design, herd management and performance testing, are presented in this paper. The foundation stock was composed of a sample of purebred heifers (66.to 71 heifers per breed) purchased at weaning in commercial herds and of semen from purebred bulls (six bulls per breed) chosen among French artificial insemination bulls. Hereford heifers and semen of bulls were added for comparison with foreign experiments. The experimental design was based on three mating phases according to generations, i.e. diallel crossing (I), crossing of purebred and F1 females with purebred males (II), crossing of F1 and 3-breed crossbred females with males from various breeds (III). In addition to comparison of genotypes, this experimental design enabled the estimation of direct and maternal additive and heterosis effects $\left(G^{I}, G^{M}, H^{I}, H^{M}\right)$. Management and performance testing were conducted in various experimental units. In spite of the duration of this experiment, the expected results remain unique.

Key words: beef cattle, French breeds, experimental design, genetic effects, lean production capacity, maternal abilities.

\section{Introduction}

Nous avons initié en 1970 une expérience de longue durée pour évaluer les aptitudes bouchères et les qualités maternelles des principales races à viande françaises en race pure et en croisement (Colleau et al., 1973). L'objet de cet article introductif est de replacer l'intérêt de cette évaluation dans le contexte de l'amélioration génétique de cette époque et de préciser l'organisation générale de l'expérience afin de faciliter la présentation et l'interprétation des différents résultats qui seront publiés dans d'autres articles.

\section{Objectif et contexte de l'expérimentation}

Face à une demande croissante de viande bovine plus maigre, la production a subi de profonds changements depuis la fin de la Seconde Guerre mondiale. Les races à viande françaises ont joué un rôle important dans ces changements en raison de leur forte croissance musculaire et de leur large diffusion (VISSAC, 1976a ; MASON \& PABST, 1977 ; MÉNISSIER, 1982a ; CUNDIFF, 1984a).

En France, un effort considérable de rationalisation et spécialisation des productions de lait et de viande a été fait à partir des années 50 dans les troupeaux bovins. La spécialisation et l'intensification de la production laitière se sont accompagnées du développement du croisement industriel avec des taureaux de races à viande spécialisées 
(Charolaise, Limousine, Blonde d'Aquitaine), voire de lignées mâles spécialisées, sur les vaches de races rustiques et de races mixtes ainsi que sur les vaches les moins productives des races laitières. Cette évolution nous a conduits à mettre en œuvre des comparaisons des principales races paternelles de croisement terminal pour une production de veaux de boucherie (VISSAC et al., 1971) puis de jeunes bovins (Frebling et al., 1967 et 1970 ; MÉnissier et al., 1982a). Par ailleurs l'effectif du cheptel de vaches allaitantes françaises produisant des veaux sevrés destinés à la production intensive de jeunes bovins s'est progressivement accru (BÉRANGER \& MÉnISSIER, 1981) sous l'effet de plusieurs phénomènes dont : (1) la reconversion vers la production de viande des troupeaux de races mixtes et surtout de races rustiques par croisement industriel ou d'absorption avec des taureaux de races à viande spécialisées ; (2) le remplacement de troupeaux laitiers par des troupeaux de races à viande spécialisées exploités en race pure ; (3) les progrès réalisés dans la maîtrise de l'alimentation des jeunes bovins notamment par une meilleure adaptation de la conduite alimentaire aux types génétiques (GEAY, 1986). Pour répondre aux implications du premier phénomène, nous avons comparé les qualités maternelles des femelles de races rustiques et croisées races à viande $\times$ races rustiques (BIBÉ et al., 1973 et 1976). Quant au second phénomène, nous ne disposions pas de comparaisons objectives des performances de nos races à viandes spécialisées exploitées en race pure. Ainsi sous l'effet de la spécialisation laitière, l'intensification et le développement de la production de viande bovine en France ont engendré une diversification du matériel génétique utilisé. Son exploitation optimale restait à étudier (MÉNISSIER et al., 1975 ; VisSAC, 1976b).

Cette période correspond également à une plus large utilisation des races continentales européennes (races à viande spécialisées françaises et italiennes, et races mixtes de grande taille) dans la plupart des pays étrangers avec des systèmes d'exploitation très diversifiés (MénISSIER, 1982a).

En Europe où dominaient les troupeaux laitiers, ce n'est qu'au début des années 70 que s'est généralisée l'introduction des races à viande françaises pour être exploitées quasi exclusivement comme races paternelles de croisement industriel. Comme en France et pour partie grâce à la coordination de la Communauté Economique Européenne (TAYLER, 1976), cette utilisation des races à viande françaises a donné lieu à de nombreuses comparaisons de races paternelles pour évaluer leur intérêt en vue d'accrồtre la production de viande des troupeaux laitiers (CASU et al., 1975 ; LiBoriusSEN, 1982 ; More O'Ferrall, 1982).

Si dans les pays traditionnels de production de viande bovine des autres continents, l'élevage en race pure ou en croisement d'absorption avec les races à viande britanniques (Angus, Hereford et Shorthorn) était le système d'expløitation dominant jusqu'aux années 50, l'utilisation du croisement et des races continentales européenpes (exotic breeds) s'est rapidement développée dès les années 60 (CundifF, 1970 ; CARTER, 1975) ; JoANDET, 1977 ; Gregory \& CUNDIFF, 1980 ; Long, 1980). En effet, les recherches engagées à partir des années 50, notamment aux Etats-Unis, ont montré l'existence d'effets d'hétérosis généralement favorables lors du croisement entre races (races à viande britanniques, races laitières ou mixtes, race Charolaise, races locales, zébus) dans la plupart des milieux de production (BiBÉ \& Foulley, 1976; Long, 1980). Par ailleurs, l'intensification de l'engraissement par une utilisątion plus importante de l'ensilage de maïs et surtout des céréales va stimuler l'intérêt des races à faible adiposité et forte croissance musculaire telles que les races continentales européennes (CundifF, 1984b). C'est alors le début de l'introduction massive des races françaises sur la plupart des continents et du développement d'un commerce international actif rendu 
possible par les progrès réalisés dans la conservation, le transport et le contrôle sanitaire du matériel génétique bovin ainsi que par la diffusion des informations sur les aptitudes de ces races obtenues dans leur système d'exploitation d'origine. Il en résulte une multitude de comparaisons de races et types génétiques réalisées avec plus ou moins de rigueur quant à la représentativité du matériel génétique évalué, l'intérêt des critères observés et le choix optimal du système de production pour évaluer les différences raciales.

La diversification du matériel génétique exploité engendre ainsi à la fin des années 60 le développement d'une approche plus rationnelle de son exploitation par une meilleure définition des objectifs d'amélioration génétique (CARTWRIGHT, 1970 et 1982a ; MÉnissier et al., 1975), par une modélisation biologique et génétique de la variabilité entre races (Dickerson, 1969, 1970 et 1973) et par le recours à l'analyse de systèmes pour optimiser l'utilisation de ce nouveau matériel génétique selon les systèmes de production (CARTwright \& Fitzhugh, 1974 ; Wilton et al., 1974 ; JoANDET \& CARTWRIGHT, 1975 ; CARTWRIGHT, 1976 et 1982b). Seul un nombre restreint d'expérimentations d'envergure seront alors poursuivies ou mises en ouvre dans les grands pays d'élevage bovin (Argentine, Canada, Etats-Unis et Nouvelle-Zélande en particulier) pour acquérir les connaissances objectives sur la variabilité génétique entre races et notamment les paramètres génétiques de croisement, nécessaires à l'exploitation rationnelle des races bovines pour la production de viande.

Ainsi dans ce contexte de la fin des années 60, les résultats acquis en France et ceux espérés de l'étranger devaient permettre d'estimer le mérite des races à viande françaises utilisées en croisement sur les principaux types biologiques maternels. Par contre la comparaison de ces races à viande françaises en races pures et l'évaluation de leur aptitude au croisement entre elles, n'étaient pas prises en compte : en France, les troupeaux de ces races étaient traditionnellement exploités en race pure dans des systèmes de production spécifiques à chacune d'elles alors qu'à l'étranger aucun pays ne disposait d'un effectif suffisant de ces races françaises pour envisager cette évaluation. Les systèmes de croisement entre races à viande étant une des voies d'amélioration de la productivité en viande du cheptel français, il était important de connaître leurs paramètres génétiques de croisement : les effets additifs et d'hétérosis directs et maternels, voire les pertes de recombinaisons épistatiques. La définition et l'interprétation de ces effets ainsi que les dispositifs expérimentaux pour les estimer, ont d'ailleurs été explicités depuis (Dickerson, 1969 et 1973 ; Rastogi \& Chevalet, 1973 ; Foulley \& LEFort, 1978 ; Dillard et al., 1980 ; Gregory et al., 1978 ; EISEN et al., 1983 ; КосH et al., 1985). C'est dans cette optique prospective que nous avons réalisé de 1970 à 1985 sous l'appellation "Vachotron », une expérience de comparaison des performances des races à viande françaises en race pure et en croisement entre elles selon un dispositif expérimental permettant d'estimer les effets additifs et d'hétérosis directs et maternels, à la fois pour les aptitudes bouchères (croissance, morphologie, efficacité alimentaire, composition corporelle, qualités des carcasses et de la viande, conditions de naissance, en particulier) et pour les qualités maternelles (évolution du poids et de la morphologie, fertilité, conditions de vêlage, allaitement et croissance des veaux jusqu'au sevrage, valeur de réforme, notamment). En raison de l'importance des moyens expérimentaux requis pour l'estimation de ces paramètres génétiques et de la taille limitée du troupeau disponible (environ 200 vaches reproductrices), l'expérimentation a été limitée à la comparaison de trois races à viande françaises. 


\section{Choix des races à évaluer et du matériel animal fondateur}

Dans le contexte ci-dessus, il a paru opportun de choisir des races de type biologique différent : deux races à viande spécialisées (Charolaise et Limousine) et une race mixte de grande taille (Maine-Anjou).

Parmi les races à viande spécialisées exploitées en France, le choix des deux premières a tenu compte de leur large diffusion en France et à l'étranger et de leur représentativité en tant que races à croissance élevée (Charolaise) ou à forte croissance musculaire (Limousine). La race Blonde d'Aquitaine a été écartée de ce choix car elle était alors exploitée en France plus comme race de croisement terminal que comme race maternelle; de plus la fréquence de l'hypertrophie musculaire d'origine génétique dans cette race (MÉnissier, 1982b) risquait d'interférer avec l'évaluation des paramètres génétiques de croisement entre ces races à viande (MÉNISSIER, 1982c).

Parmi les races mixtes de grande taille, nous disposions en France des races MaineAnjou (région Ouest) et Pie-Rouge de l'Est (région Est). La race Maine-Anjou a été choisie en raison de son utilisation croissante dans les troupeaux allaitants français en race pure et en croisement, alors que la race Pie-Rouge de l'Est était presque exclusivement exploitée dans des troupeaux laitiers traits et en race pure.

Pour chacune des trois races françaises retenues, le matériel animal fondateur $\left(G_{o}\right)$ de l'expérimentation a été constitué, d'une part, par un échantillon de génisses reproductrices de race pure nées en 1970 (66 à 71 génisses par race) et achetées au sevrage dans des élevages français de production et, d'autre part, par la semence de taureaux de race pure (6 taureaux par race) choisis parmi ceux des centres français de production de semence (tableau 1).

L'échantillonnage des génisses, réalisé par une seule personne et indépendamment de leur type morphologique, a été pratiqué au moment du sevrage avant qu'intervienne une quelconque sélection des éleveurs pour la mise à la reproduction; seules les génisses de poids et conformation extrêmes ont été écartées du choix. Ce choix s'est opéré dans un grand nombre d'élevages situés dans les principaux départements des berceaux d'origine de ces races et représentatifs de la diversité de leurs systèmes d'exploitation. Ainsi, les génisses Charolaises provenaient à la fois de la zone où dominait traditionnellement la production de châtrons partir d'un cheptel de grand format (Allier) et de celle où la sélection était plus orientée vers la production de bovins plus jeunes et à meilleure conformation bouchère (Sâone-et-Loire, Loire). Par contre, la zone Ouest (Vendée) où la pratique ancienne de la traite liée au métayage aurait pu favoriser l'aptitude laitière, a été écartée.

Près de la moitié des génisses Limousines ont été achetées dans des petits élevages (Corrèze) produisant traditionnellement des veaux de boucherie ou des jeunes bovins « à l'auge » à partir d'un cheptel sélectionné sur la conformation bouchère et la croissance dans le jeune âge, et généralement exploité par insémination artificielle. L'autre moitié provenait de la zone (Haute-Vienne, voire Indre) où dominaient les élevages de plus grande taille pratiquant le «plein air », utilisant plutôt la monte naturelle et produisant des bovins plus âgés avec un cheptel davantage sélectionné sur la croissance, la taille voire la fertilité des mères.

Pour les génisses Maine-Anjou, c'est surtout la variation de densité du cheptel de cette race qui a conduit à la répartition du choix selon les départements d'origine. Ces 
TABLEAU 1

Caractéristiques des génisses et taureaux fondateurs selon la race

Characteristics of foundation heifers and bulls according to breeds

\begin{tabular}{|c|c|c|c|c|}
\hline Race & Charolaise & Limousine & Maine-Anjou & Hereford \\
\hline - Génisses $\left(\mathrm{G}_{\mathrm{O}}\right)$ : & & & & \\
\hline Effectif $\ldots \ldots$ & 71 & 66 & 69 & 25 \\
\hline $\begin{array}{l}\text { Date moyenne de naissance } \\
\text { (étendue) } \ldots \ldots \ldots \ldots \ldots \ldots\end{array}$ & 2 mars 1970 (99 j.) & 3 mars $1970(72 \mathrm{j})$. & 27 février $1970(93 \mathrm{j})$. & 15 avril 1970 (93 j.) \\
\hline $\begin{array}{l}\text { Nombre de pères } \\
\text { (dont insémination artificielle) } \ldots\end{array}$ & $9(7)$ & $12(7)$ & $29(4)$ & $?$ \\
\hline $\begin{array}{l}\text { Nombre moyen de génisses par } \\
\text { pères (étendue) } \ldots \ldots \ldots \ldots \ldots\end{array}$ & $7,8(1-10)$ & $5,5(3-10)$ & $2,4(1-9)$ & - \\
\hline $\begin{array}{l}\% \text { de génisses nées d'insémination } \\
\text { artificielle } \ldots \ldots \ldots \ldots \ldots \ldots\end{array}$ & $85,9 \%$ & $50,0 \%$ & $20,3 \%$ & - \\
\hline Nombre d'élevages $\ldots \ldots \ldots \ldots$. & 50 & 38 & 32 & 10 \\
\hline $\begin{array}{l}\text { Nombre moyen de génisses par éle- } \\
\text { vage (étendue) } \ldots \ldots \ldots \ldots \ldots \ldots\end{array}$ & $1,4(1-10)$ & $1,7(1-10)$ & $2,2(1-5)$ & $2,5(1-6)$ \\
\hline$\%$ de génisses par région (1) . . . . & $\begin{array}{l}« 71 "=49,3 \% \\
" 03 "=43.7 \% \\
« 42 "=7.0 \%\end{array}$ & $\begin{array}{l}« 19 »=50,0 \% \\
« 87 »=42,4 \% \\
« 36 »=7,6 \%\end{array}$ & $\begin{array}{l}« 49 »=29,0 \% \\
« 79 »=26,1 \% \\
« 53 »=23,2 \% \\
" 44 »=17,4 \% \\
« 72 »=4,3 \%\end{array}$ & $\begin{array}{c}\text { "Canada- } \\
\text { Ontario } "=100 \%\end{array}$ \\
\hline \multicolumn{5}{|l|}{ - Taureaux $\left(\mathrm{G}_{\mathrm{o}}\right)$ : } \\
\hline Effectif utilisé $\ldots \ldots \ldots \ldots \ldots$ & 6 & 6 & 6 & 6 \\
\hline $\begin{array}{l}\text { Poids moyen de naissance de leurs } \\
\text { veaux en race pure }(2) \text { : }\end{array}$ & & & & (3) \\
\hline - ensemble des taureaux $\ldots \ldots$ & $45,0 \mathrm{~kg}$ & $38,2 \mathrm{~kg}$ & $49,5 \mathrm{~kg}$ & $(5,53 \mathrm{pts})$ \\
\hline - taureaux extrêmes . . . . . . . & $42,5-47,4 \mathrm{~kg}$ & $35,9-40,6 \mathrm{~kg}$ & $48,3-50,6 \mathrm{~kg}$ & $(4,95-5,92 \mathrm{pts})$ \\
\hline naissances faciles et les 4 autres ... & $-3,2 \mathrm{~kg}$ & $-3,2 \mathrm{~kg}$ & $-1,2 \mathrm{~kg}$ & $(+0,29 \mathrm{pts})$ \\
\hline
\end{tabular}

(1) Départements ; districts : “ 03 » Allier, « 19 » Corrèze, « 36 » Indre, « 42 » Loire, « 44 » Loire-Atlantique, " 49 " Maine-et-Loire, « 53 » Mayenne, « 71 " Saône-et-Loire, « 72 " Sarthe, « 79 » Deux-Sèvres, « 87 » HauteVienne.

(2) Données obtenues dans les éleviages commerciaux, pour les taureaux de races françaises ; Data from commercial herds, for bulls of French breeds.

(3) Note croissante (1 à 6) de facilité de naissance en troupeau expérimental pour les taureaux Hereford ; Score (I to 6) of easy birth in experimental herd for Hereford bulls.

zones pratiquaient des systèmes extrêmement diversifiés tant entre qu'intra troupeaux voire durant la carrière d'une même vache : troupeaux traits (Mayenne et Sarthe), troupeaux ne pratiquant la traite que sur une partie des vaches ou des lactations (Maine-et-Loire, Deux-Sèvres, voire Loire-Atlantique).

Cette diversité des systèmes d'élevage entre races sera à considérer pour l'interprétation des différences entre races notamment pour les performances des génisses fondatrices jusqu'à leur mise à la reproduction. De plus, la représentativité de l'échantillonnage des génisses intra race a été assurée en limitant le nombre de génisses issues d'un même père tout en recherchant la constitution de familles paternelles en vue d'une 
interprétation éventuelle des effets grand-paternels. Du fait des variations de pénétration de l'insémination artificielle et de taille des troupeaux dans ces races, le nombre de familles paternelles et la fréquence des génisses issues d'insémination artificielle ont été assez différents selon les races (tableau 1).

Les taureaux fondateurs ont été choisis parmi ceux exploités en France pour l'insémination artificielle en race pure et agréés sur descendance pour la production de bovins de boucherie selon les systèmes en vigueur dans chaque race. Il s'agissait de taureaux encore vivants en 1971 et ayant une semence de bonne qualité. Pour faciliter la gestion et l'utilisation des semences, le choix a été limité à six taureaux par race. Le choix intra race s'est opéré en fonction de la répartition des poids moyens de naissance des veaux de race pure engendrés par ces taureaux dans les élevages commerciaux, afin de ne retenir qu'un taureau par sextile de cette distribution, et notamment le taureau considéré par les utilisateurs comme ayant les meilleures aptitudes bouchères. Les poids moyens à la naissance des veaux engendrés par les taureaux choisis (tableau 1) correspondent assez bien à ceux habituellement observés dans chacune de ces races (MÉNISSIER \& Foulley, 1979). Les six taureaux Limousins étaient indexés sur descendance pour la production de veaux (cinq) ou de jeunes bovins (un) de boucherie et possédaient en moyenne une valeur génétique supérieure à celle de leurs contemporains pour la croissance et la conformation bouchère $(+3,5$ et $+1,8 \%$ de la moyenne respectivement). Les taureaux Charolais étaient évalués essentiellement sur la taille et la morphologie de leurs descendants au sevrage en ferme et pour trois d'entre eux sur les qualités maternelles de leurs filles en station. Pour la croissance et la conformation bouchère, leur valeur génétique peut être considérée comme représentative de la moyenne des taureaux d'insémination artificielle utilisés en race pure au début des années 70 . Quant aux taureaux Maine-Anjou, ils étaient parmi ceux jugés par les utilisateurs comme produisant des bovins de valeur bouchère supérieure à la moyenne parmi ceux contrôlés sur descendance pour la production laitière.

Par ailleurs, bien que n'existant pratiquement pas dans les élevages français, la race Hereford a été ajoutée à cette évaluation des races françaises (évaluation en race pure uniquement) afin de servir de connexion entre cette expérimentation et les essais étrangers utilisant largement cette race comme référence. Nous avons importé du Hereford nord-américain puisqu'il était réputé sélectionné sur la croissance et les qualités maternelles. La semence des six taureaux fondateurs a été choisie selon des principes équivalents à ceux retenus pour les races françaises, en considérant les facilités de naissance à défaut du poids à la naissance et le poids des veaux au sevrage pour les aptitudes bouchères. Ce choix s'est opéré parmi les taureaux disponibles dans le troupeau expérimental de l'Université du Colorado (San Juan Basin Branch, Colorado Experiment Station, Hesperus, USA) utilisé pour la création de lignées consanguines et la sélection sur la croissance. Ces taureaux appartenaient à cinq lignées différentes dont les caractéristiques paternelles et maternelles ont été évaluées depuis (MANGus \& BRINKs, 1971 ; Hohenboken \& Brinks, 1971 ; Brinks et al., 1973 ; Hays \& Brinks, 1980). Du fait de difficultés techniques, les 25 génisses fondatrices Hereford n'ont pu être importées des Etats-Unis. Elles ont été importées de 10 élevages commerciaux de l'Ontario (Canada) sans critère de choix zootechnique ; ce qui explique qu'elles sont nées en moyenne 1,5 mois plus tard que les génisses de races françaises (tableau 1). 


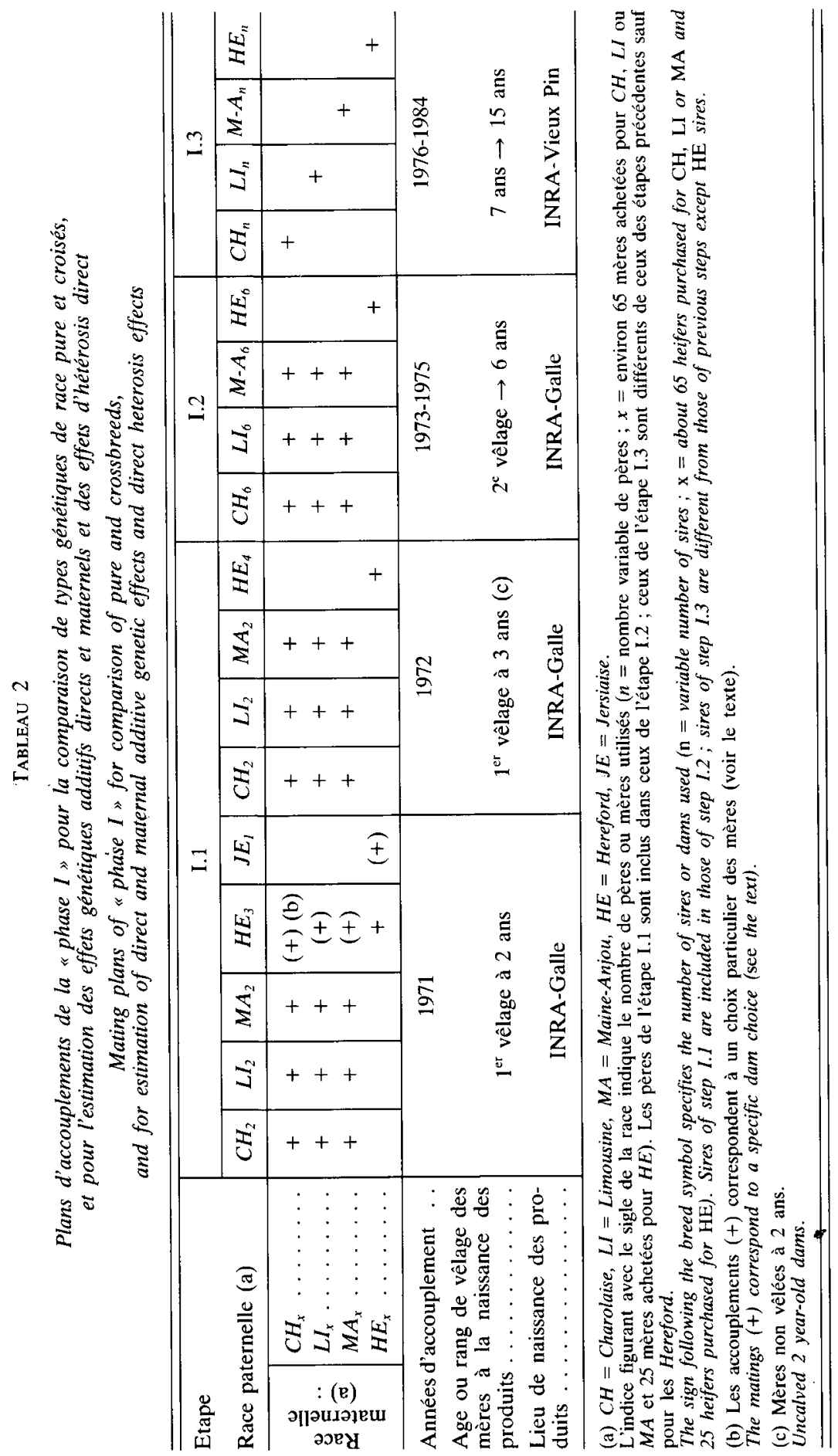




\section{Dispositif expérimental utilisé}

L'expérience a été planifiée en trois phases successives et imbriquées correspondant aux différents systèmes d'accouplements sur trois générations $\left(G_{0}\right.$ à $G_{2}$ pour les parents, $G_{1}$ à $G_{3}$ pour les descendants).

\section{A. Phase I: estimation des effets génétiques additifs directs et maternels et des effets d'hétérosis directs (tableau 2)}

Cette phase a été réalisée par accouplements des pères et mères fondateurs $\left(G_{0}\right)$ selon un plan de croisement diallèle complet entre les trois races françaises afin de procréer des produits $\left(G_{1}\right)$ de 9 types génétiquas différents. Par contre, les mères Hereford ont été accouplées uniquement en race pure. Cette première phase s'est déroulée en trois étapes selon l'âge et le rang de vêlage des mères (tableau 2). Le dispositif d'accouplements ci-dessus a été strictement appliqué après le premier vêlage des mères et jusqu'à leur mise à la reproduction à l'âge de 5 ans (étape I.2). La pratique d'un premier vêlage précoce étant un des moyens envisagés pour accroître la productivité des troupeaux à viande (TAYLER, 1975) et afin d'être dans les conditions optimales d'évaluation des difficultés de naissance, nous avons choisi de pratiquer un premier vêlage à 2 ans. Cependant pour le premier vêlage à 2 ans ainsi que pour le premier vêlage à 3 ans des génisses n'ayant pu être fécondées pour vêler à 2 ans (étape I.1), en raison des risques trop importants de difficultés de vêlage et de leurs conséquences sur la carrière ultérieure des mères, seul un nombre limité de pères (deux pour chaque race française, trois à quatre pour la race Hereford) choisis pour leurs facilités de naissance évaluées antérieurement (tableau 1) ont été utilisés. Pour le premier vêlage à 2 ans, environ $1 / 5^{\mathrm{e}}$ des génisses fondatrices de chaque race a été écarté du plan d'accouplements prévu, compte tenu d'un développement corporel et d'une ouverture pelvienne rapportée au poids jugés insuffisants avant leur mise à la reproduction. Ces femelles écartées pour le premier vêlage du plan diallèle de croisement ont été accouplées à des taureaux de races de plus petite taille : Hereford pour celles de races françaises et Jersiaise pour celles de race Hereford. Pour les vêlages ultérieurs, elles ont été replacées dans le dispositif expérimental décrit précédemment. Les caractéristiques de ce choix des génisses et l'efficacité de celui des pères ont été rapportées par Ménissier et al. (1974). Les produits ainsi engendrés ont été, pour les femelles, conservés pour la reproduction (phase II) et, pour les mâles, conduits pour une production de taurillons abattus à 15 ou 18 mois. A partir de l'âge de 6 ans (étape I.3), les accouplements des vaches ont été réalisés uniquement en race pure. Les vaches ont alors été exploitées pour évaluer leur longévité et comparer les aptitudes bouchères de leurs produits mâles de race pure dans un système de production de bœufs de 30 mois (Frebling et al., 1980 et 1982 ; Gillard et al., 1986).

A partir des effets estimés pour les neufs types génétiques impliquant les races françaises (trois races pures et six croisements F1, étapes I.1 et I.2), il est possible d'estimer les effets génétiques directs $\left(G^{I}\right)$ et maternels $\left(G^{M}\right)$ ainsi que les effets spécifiques d'hétérosis direct $\left(H^{I}\right)$ pour les trois races françaises. En effet, selon les 'définitions et notations proposées par Dickerson (1973), chacun des effets de ces neufs types génétiques $\left(X_{i j}\right.$, le premier indice représentant l'indice de la race paternelle et le 


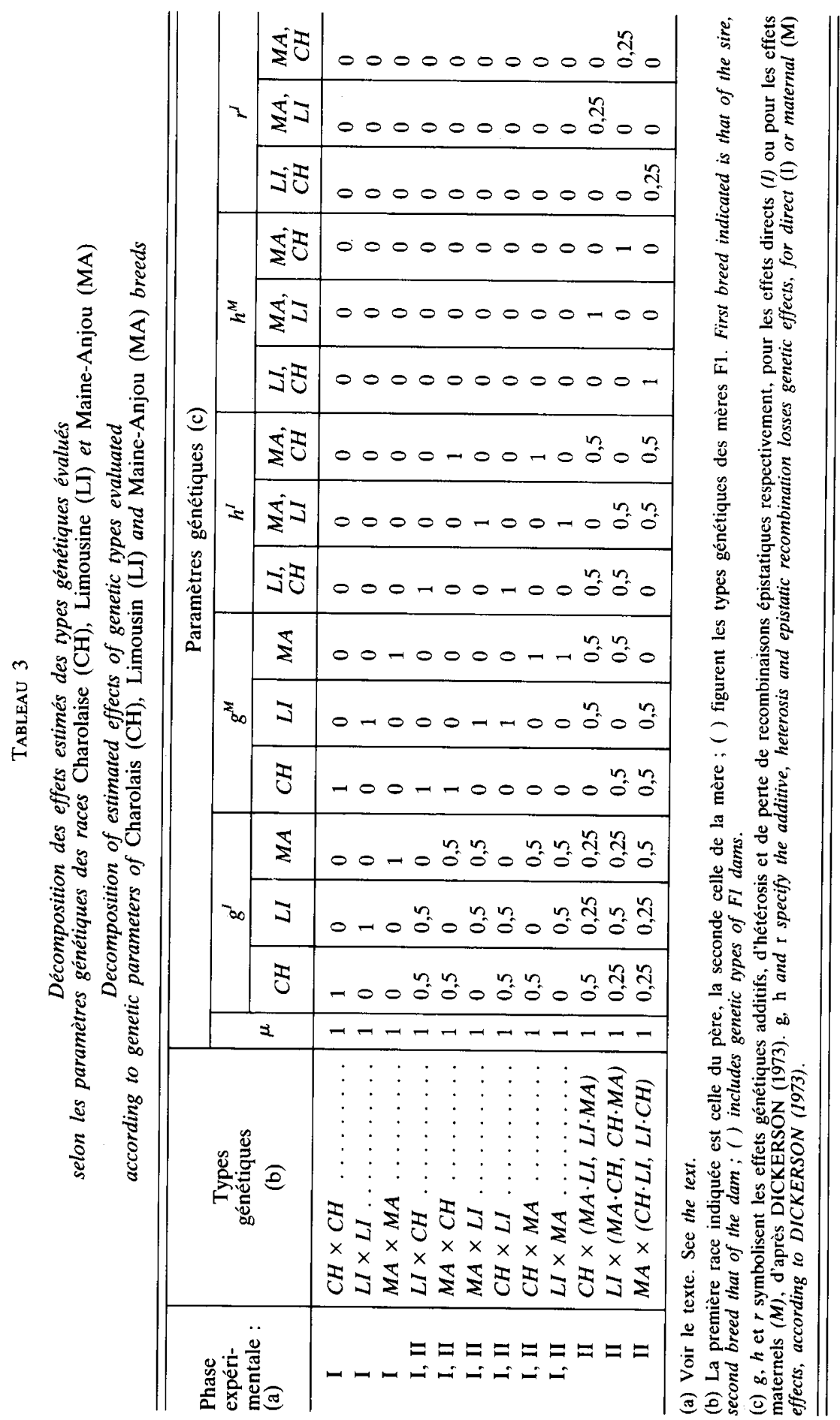


second indice celui de la race maternelle) correspond à une combinaison des principaux effets génétiques (tableau 3). La résolution de ce système d'équations (Foulley \& LEFORT, 1978 ; GREGORY et al., 1978) conduit aux solutions suivantes :

$\hat{\mu}=1 / 3 \sum_{i=1}^{3} X_{i i}$, qui représente la moyenne des races pures ;

$\hat{g}^{M}{ }_{i}=1 / 3 \sum_{j=1}^{3}\left(X_{j i}-X_{i j}\right)$, avec $\sum_{i=1}^{3} \hat{g}^{M}{ }_{i}=0$;

$\hat{g}_{i}^{\prime}=X_{i i}-\hat{\mu}-\hat{g}_{i}^{M}$, avec $\sum_{i=1}^{3} \hat{g}_{i}^{I}=0$;

$\hat{h}_{i j}^{I}=1 / 2\left(X_{i j}+X_{j i}-X_{i i}-X_{j j}\right)$, en admettant que $\hat{\mathrm{h}}_{i j}^{I}=\hat{\mathrm{h}}_{j i}^{I}$.

Les effets maternels additifs ainsi estimés incluent les effets maternels de dominance (EISEN et al., 1983) s'ils existent, et supposent l'égalité des niveaux génétiques des pères et mères fondateurs d'une même race (Foulley \& LeForT, 1978). Si cette dernière hypothèse n'était pas admise, seuls les effets moyens des trois races pures et leurs effets spécifiques d'hétérosis direct seraient estimables. Par ailleurs, d'autres combinaisons des effets des types génétiques sont également estimables en raison de leur intérêt zootechnique (effets des races paternelles, des races maternelles, d'hétérosis direct moyen par race, etc.), en sachant que généralement leurs estimations ne sont pas statistiquement indépendantes et leurs tests de signification inappropriés (GREGORY et al., 1978).

Lors de cette phase, les aptitudes évaluées concernent à la fois les caractères maternels des femelles fondatrices $\left(G_{0}\right)$ des quatre races pures de leur sevrage à leur réforme, et les performances des descendants $\left(G_{1}\right)$ de races pures et croisés de leur gestation à leur sevrage pour les mâles et les femelles, durant leur engraissement et à l'abattage pour les mâles, de leur sevrage à l'âge de 18 mois pour les femelles.

\section{B. Phase II : estimation des effets d'hétérosis maternel}

et comparaison des mères de race pure et croisées $F 1$ et de leurs produits croisés (tableau 4)

Cette seconde phase a été réalisée à partir des mères $\left(G_{1}\right)$ de race pure et croisées F1 procréées lors de la phase précédente, en vue de disposer de produits $\left(G_{2}\right)$ de types génétiques permettant à la fois l'estimation des effets spécifiques d'hétérosis maternel $\left(h^{M}\right)$ et la comparaison des types génétiques croisés trois races. La phase II s'est déroulée en trois étapes successives (tableau 4).

Plusieurs dispositifs expérimentaux étaient possibles (Foulley \& LEForT, 1978) pour l'étape principale (II.2). Compte tenu des difficultés pratiques rencontrées pour disposer de semence de taureaux croisés et du souhait de comparer des produits croisés trois races, le dispositif d'accouplements retenu a été de procréer des croisés deux races (six types génétiques $\mathrm{F} 1$ en distinguant les croisements réciproques) et des croisés trois races (six types génétiques en distinguant les mères de croisements réciproques) respectivement issus de mères de race pure accouplées en croisement avec des pères de race pure des deux autres races et issus de mères croisées $F 1$ accouplées à des pères de race pure différente de celles présentes chez la mère F1. Les mères Hereford ont été accouplées en race pure. Tous les pères utilisés étaient les mêmes que ceux employés dans la phase I (I.1 et I.2). 


\section{TABLEAU 4}

Plans d'accouplements de la "phase II» pour l'estimation des effets d'hétérosis maternel et la comparaison des mères de races pures et croisées FI

Mating plans of «phase II» for estimation of maternal heterosis effects and for comparison of pure- and crossbred dams

\begin{tabular}{|c|c|c|c|c|c|c|c|c|c|c|}
\hline \multirow{2}{*}{\multicolumn{2}{|c|}{$\begin{array}{l}\text { Etape } \\
\text { Race paternelie (a) }\end{array}$}} & \multicolumn{3}{|c|}{ II.1 } & \multicolumn{4}{|c|}{ II. 2} & \multicolumn{2}{|c|}{ II. 3} \\
\hline & & $\frac{L I_{3}}{+}$ & $\begin{array}{c}A N_{3} \\
+\end{array}$ & $\begin{array}{r}J E_{3} \\
+\end{array}$ & $\mathrm{CH}_{6}$ & $\frac{L I_{6}}{+}$ & $\frac{M A_{6}}{+}$ & $H E_{6}$ & $\begin{array}{c}B A_{4} \\
+\end{array}$ & $P E_{4}$ \\
\hline 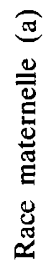 & $\begin{array}{l}C H_{x} \ldots \ldots \ldots \\
L I_{x} \ldots \ldots \ldots \\
M A_{x} \ldots \ldots \ldots \ldots \\
H E_{x} \ldots \ldots \ldots \ldots \\
M A \times C H_{x} \text { ou } C H \times M A_{x} \\
M A \times L I_{x} \text { ou } L I \times M A_{x} \ldots \\
C H \times L I_{x} \text { ou } L I \times C H_{x} \ldots\end{array}$ & $\begin{array}{l}+ \\
+ \\
+ \\
+ \\
+ \\
+ \\
+\end{array}$ & $\begin{array}{l}+ \\
+ \\
+ \\
+ \\
+ \\
+ \\
+\end{array}$ & $\begin{array}{l}+ \\
+ \\
+ \\
+ \\
+ \\
+ \\
+\end{array}$ & $\begin{array}{l}+ \\
+\end{array}$ & $\begin{array}{l}+ \\
+\end{array}$ & $\begin{array}{l}+ \\
+\end{array}$ & + & $\begin{array}{l}+ \\
+ \\
+ \\
+ \\
+ \\
+ \\
+\end{array}$ & $\begin{array}{l}+ \\
+ \\
+ \\
+ \\
+ \\
+ \\
+\end{array}$ \\
\hline \multicolumn{2}{|c|}{ Années d'accouplement $\ldots \ldots \ldots$} & \multicolumn{3}{|c|}{ 1973-1977 } & \multicolumn{4}{|c|}{$1974-1983$} & \multirow{2}{*}{\multicolumn{2}{|c|}{$\begin{array}{l}1980-1983 \\
9 \text { à } 11 \text { ans }\end{array}$}} \\
\hline \multicolumn{2}{|c|}{$\begin{array}{l}\text { Age des mères à la naissance des } \\
\text { produits } \ldots \ldots \ldots \ldots \ldots \ldots\end{array}$} & \multicolumn{3}{|c|}{2 ans } & \multicolumn{4}{|c|}{3 à 8 ans } & & \\
\hline \multicolumn{2}{|c|}{ Lieu de naissance des produits . . . } & \multicolumn{3}{|c|}{ INRA-Galle } & \multicolumn{4}{|c|}{ INRA-Galle } & \multicolumn{2}{|c|}{ INRA-Galle } \\
\hline
\end{tabular}

(a) $\mathrm{CH}=$ Charolaise, $\mathrm{LI}=$ Limousine, $\mathrm{MA}=$ Maine-Anjou, $\mathrm{HE}=$ Hereford, $\mathrm{AN}=$ Angus, $\mathrm{JE}=$ Jersiaise, $\mathrm{BA}=$ Blonde d'Aquitaine, $\mathrm{PE}=$ Pie-Rouge de l'Est. L'indice figurant avec le sigle de chaque race indique le nombre de pères et mères utilisés. Les pères de l'étape II.2 sont les mêmes que ceux de la phase I (étapes: I.1 et I.2) ; les pères $L I$ et $J E$ (II.1) sont différents de ceux de l'étape II.2 ou de la phase I; $x=$ environ 30 génisses par type génétique provenant de la phase I (étapes I.1 et I.2) et nées de 1972 à 1976.

The sign following the breed symbol specifies the number of sires or dams used. Sires in step II.2 are the same as those of steps 1.1 and 1.2 (phase I); LI and $\mathrm{JE}$ sires are different from step II.2 and phase $I ; \mathrm{x}=$ about 30 heifers per genetic type from phase I (steps 1.1 and I.2) and born between 1972 and 1976.

En plus des comparaisons des effets de ces différents types génétiques, il est possible d'estimer les effets spécifiques d'hétérosis maternel $\left(h^{M}{ }_{i j}\right)$ pour les trois races françaises évaluées à partir de la décomposition de ces effets des types génétiques $\left(X_{k j i}\right.$, les indices $k, i, j$, représentant respectivement ceux de la race du père, du grand-père maternel et de la grand-mère maternelle) en effets génétiques directs et maternels (tableau 3) :

en admettant que $\hat{h}_{i j}^{M}=\hat{h}^{M}{ }_{i i}$.

$$
\hat{h}^{M}{ }_{i j}=1 / 2\left(X_{k i j}+X_{k j i}-X_{k i i}-X_{k j j}\right),
$$

Ces estimations des effets spécifiques d'hétérosis maternel contiennent aussi une partie des effets directs liés aux pertes de recombinaisons épistatiques $\left(1 / 4 \cdot r_{i j}^{\prime}\right.$ Foulley \& LEFORT, 1978). Par ailleurs, l'analyse conjointe des phases I (étapes I.1 et I.2) et II (étape II.2) fournira une estimation simultanée de l'ensemble des effets génétiques concernant les trois races françaises $\left(g^{I}, g^{M}, h^{I}\right.$ et $\left.h^{M}\right)$. Les estimations des effets d'hétérosis direct seront plus précises et la conformité à l'hypothèse d'égalité des niveaux génétiques des pères et des mères de même race, sera plus acceptable pour estimer les effets maternels additifs. 
Pour le premier vêlage à deux ans, afin de réduire les difficultés de naissance et de comparer l'intérêt de races paternelles de petite taille pour maîtriser ces difficultés (MÉnissier et al., 1975 et 1982b, MÉNissier, 1975a), les mères ont été accouplées à des taureaux (trois taureaux par race paternelle) de races de types biologiques différents (étape II.1): une race laitière spécialisée (Jersiaise), une race à viande spécialisée britannique (Angus) et une race à viande spécialisée française (Limousine). Le choix des taureaux intra race s'est fait différemment selon leur race en vue de réduire les différences entre celles-ci pour certains caractères : les taureaux Jersiais ont été choisis en raison de la plus grande taille de leurs filles; les taureaux Angus ont été proposés par le Milk Marketing Board (Royaume-Uni) au vu de leurs meilleures aptitudes bouchères (poids individuel à un an et contrôle sur descendance en station) et de leur réputation pour les facilités de naissance en croisement ; les taureaux Limousins ont été sélectionnés parmi ceux agréés pour l'insémination artificielle et ayant produit les veaux les plus légers lors de leur contrôle sur descendance en ferme. Par rapport à la moyenne de leurs contemporains d'évaluation, ces taureaux Limousins possèdaient une valeur génétique inférieure pour le poids de naissance $(-1 \%$ environ) et la croissance ( $-2 \%$ environ) mais supérieure pour la conformation bouchère $(+8 \%$ environ).

Les mères $G_{1}$ ayant terminé l'étape principale d'évaluation (étape II.2) et âgées de plus de 8 ans ont été accouplées jusqu'à l'achèvement de l'expérience (étape II.3) à deux races paternelles (Blonde d'Aquitaine et Pie-Rouge de l'Est) différentes des trois races françaises évaluées. Le choix de ces races s'est opéré en fonction de la nécessité, d'une part, de procréer des veaux à fort potentiel de croissance pour différencier de façon optimale les qualités maternelles des mères de race pure et croisées $F 1$, et, d'autre part, d'évaluer des races paternelles de croisement autres que celles déjà utilisées mais appartenant aux mêmes types biologiques (races françaises mixte de grande taille ou à viande spécialisée). Le choix des taureaux (quatre taureaux par race) a été fait parmi ceux d'insémination artificielle très utilisés par les éleveurs français. Les taureaux Pie-Rouge de l'Est ont été choisis sur leur réputation à engendrer des produits à bonnes aptitudes bouchères parmi ceux sélectionnés pour la production laitière. Les taureaux Blonds d'Aquitaine ont été sélectionnés pour leurs performances bouchères contrôlées sur descendance en croisement (production de veaux de boucherie ou de jeunes bovins).

Avec ce dispositif particulier d'accouplements des mères pour leur $1^{\text {er }}$ vêlage (étape II.1) et leur fin de carrière (étape II.3), les effets spécifiques d'hétérosis maternel pour les trois races françaises Charolaise, Limousine et Maine-Anjou peuvent être estimés comme pour la seconde étape décrite antérieurement (II.2) à partir des effets des types génétiques maternels ajustés pour les effets des races paternelles. Par contre l'interprétation des autres comparaisons entre types génétiques maternels ou paternels en fonction des paramètres génétiques de ces races est partiellement modifiée puisque les races paternelles sont différentes des races maternelles. Outre les différences dues aux effets génétiques additifs des races impliquées dans ces comparaisons, sont inclus en proportion variable les écarts d'effets d'hétérosis direct spécifique entre les races des types génétiques maternels et les races paternelles.

Durant cette seconde phase, les aptitudes évaluées sur les mères $\left(G_{1}\right)$ et les produits $\left(G_{2}\right)$ ainsi procréés sont pour la plupart identiques à celles décrites pour la phase I. 
C. Phase III : comparaison de divers types génétiques paternels et maternels (tableaux 5, 6 et 7)

Cette dernière phase expérimentale correspond en fait à une série de comparaisons de divers types génétiques croisés, utilisant les génisses $G_{2}$ procréées lors de la phase II ; sa finalité étant de fournir des informations préliminaires sur les potentialités de types génétiques généralement peu utilisés en France et exploitables dans des systèmes d'élevages spécifiques. Ces comparaisons ont été organisées en trois étapes en fonction de leur objectif principal.

\section{Production de génisses unipares (étape III.1 - tableau 5)}

Une des limites au croisement sur génisses de races à viande françaises avec des races paternelles de petite taille pour réduire leurs difficultés de vêlage, est la valorisation commerciale des produits femelles croisés (MÉNISSIER, 1975a et 1975b; MÉNISSIER et al., 1975). Un des systèmes de valorisation proposé durant les années 70 était de les abattre comme des jeunes bovins après un $1^{\text {er }}$ vêlage précoce (" génisses unipares " Petit et al., 1973 ; Petit, 1975). Ainsi, les génisses croisées de pères Limousins, Angus et Jersiais issus de l'étape II.1 ont été exploitées selon ce système pour comparer les

\section{TABLEaU 5}

Plans d'accouplements de la "phase III" pour la comparaison de trois types génétiques maternels en production de génisses unipares

Mating plans of "phase III " for comparison between three maternal genetic types for oncebred heifer production

\begin{tabular}{|c|c|c|}
\hline \multirow{2}{*}{\multicolumn{2}{|c|}{$\begin{array}{l}\text { Etape } \\
\text { Race paternelle (a) }\end{array}$}} & III.1 \\
\hline & & $B A_{3}$ (b) \\
\hline 导 & 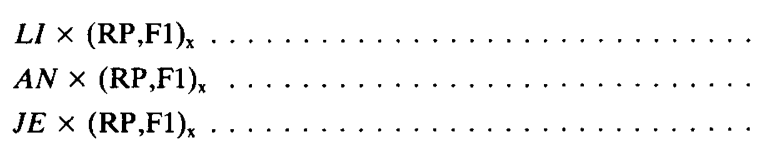 & $\begin{array}{l}+ \\
+ \\
+\end{array}$ \\
\hline \multicolumn{2}{|c|}{ 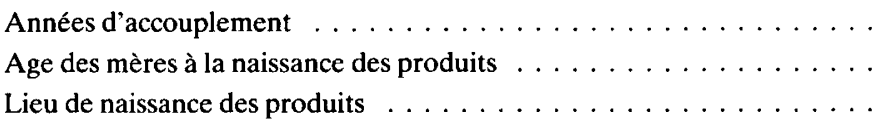 } & $\begin{array}{l}\text { 1975-1979 } \\
2 \text { ans } \\
\text { INRA-La Minière }\end{array}$ \\
\hline \multicolumn{3}{|c|}{ 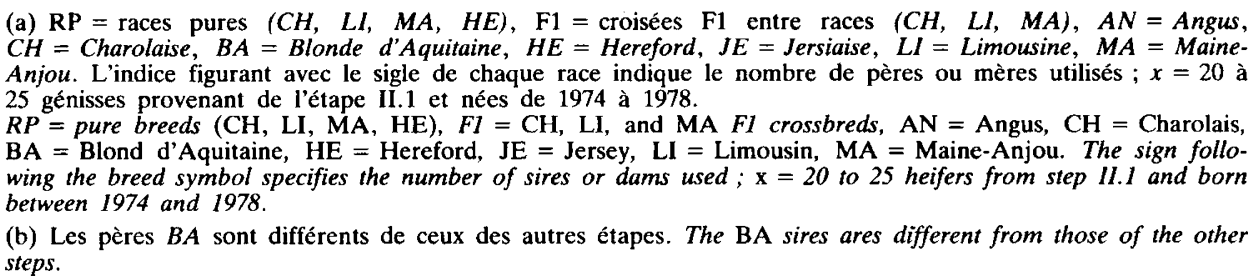 } \\
\hline
\end{tabular}


performances de ces trois races (MÉNISSIER et al., 1982b). Elles ont été accouplées avec une race à viande spécialisée (Blonde d'Aquitaine) différente de celles des types génétiques maternels. Les trois pères Blonds d'Aquitaine, différents de ceux des autres étapes expérimentales, ont été choisis parmi les taureaux d'insémination artificielle contrôlés sur descendance en croisement pour la production de veaux de boucherie, en raison de leur faible poids de naissance et de leur supériorité de conformation bouchère (valeur génétique de $-5,-4$ et $+19 \%$ de la moyenne du lot témoin racial pour respectivement le poids à la naissance, la croissance et la conformation bouchère). Les aptitudes évaluées ont concerné la valeur bouchère et la fertilité des femelles primipares $\left(G_{2}\right)$ et les conditions de naissance de leurs veaux $\left(G_{3}\right)$.

\section{Productivité des vaches croisées trois races à viande (étape III.2 - tableau 6)}

Le croisement rotatif et la création de races synthétiques sont des voies possibles pour améliorer la productivité des troupeaux à viande (Cundiff et al., 1986). Ainsi à partir des génisses de races pures procréées lors de l'étape I.3 et des génisses croisées trois races issues de l'étape II.2, nous avons initié une comparaison des performances des vaches de race pure et croisées trois races (Charolaise, Limousine et Maine-Anjou). Pour limiter l'effectif mis en comparaison, les femelles croisées trois races possèdant

\section{Tableau 6}

Plans d'accouplements de la "phase III" pour la comparaison de types génétiques maternels croisés 3 races à viande Mating plans of "phase III" for comparison between maternal 3-breed crossbred genetic types

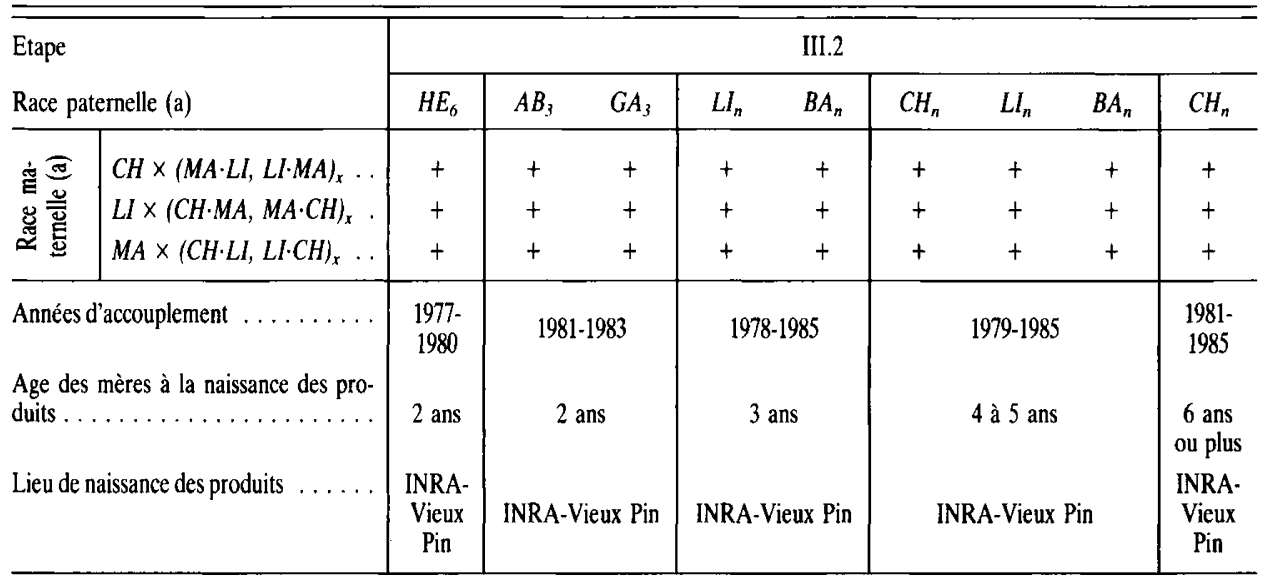

(a) $C H=$ Charolaise,$L I=$ Limousine, $M A=$ Maine-Anjou, $H E=$ Hereford, $B A=$ Blonde d'Aquitaine, $A B=$ Abondance, $G A=$ Gasconne. L'indice figurant avec le sigle de chaque race indique le nombre de pères ou mères utilisés $(n=$ nombre variable de pères, $x=50$ à 70 génisses provenant de l'étape II.2 nées de 1976 à 1982). Les pères des races à viande spécialisées françaises sont pour partie identiques à ceux de l'étape I.3 mais différents des autres étapes. Les pères $H E$ sont différents de ceux des phases I et II.

$\mathrm{CH}=$ Charolais, $\mathrm{LI}=$ Limousin, $\mathrm{MA}=$ Maine-Anjou, $\mathrm{HE}=$ Hereford, $\mathrm{BA}=$ Blond d'Aquitaine, $\mathrm{AB}=\mathrm{Abon}-$ dance, $\mathrm{GA}=\mathrm{Gascon}$. The sign following the breed symbol specifies the number of sires or dams used $(\mathrm{n}=$ variable number of sires, $\mathrm{x}=50$ to 70 heifers from step 11.2 and born between 1976 and 1982). The sires from French specialized beef breeds are almost the same as those of step 1.3, but different from those of the other steps. HE sires are different from those of phases $I$ and $I I$. 
une trop faible taille et un développement musculaire trop faible ou trop important, ont été éliminées avant la mise à la reproduction (1/3 environ). Les accouplements ont été pratiqués avec des taureaux de races de taille croissante selon l'âge des mères (tableau 6), choisies en fonction de leur intérêt zootechnique pour maîtriser les difficultés de naissance et pour procréer des veaux destinés aussi bien au renouvellement (femelles) qu'à l'engraissement (mâles). Les taureaux Hereford provenaient du Royaume-Uni. Les taureaux Angus, différents de ceux de l'étape II.1, ont été sélectionnés parmi ceux du Milk Marketing Board (Royaume-Uni) pour leur supériorité d'aptitudes bouchères et de facilités de naissance contrôlées sur descendance en croisement. Les taureaux de race Abondance ont été retenus d'après leur réputation auprès des éleveurs français pour engendrer des veaux à bonnes aptitudes bouchères en race pure. Les taureaux de races à viande françaises différents de ceux utilisés pour les autres étapes, ont été choisis pour leur supériorité d'aptitudes bouchères et de qualités maternelles évaluées lors de leur contrôle sur descendance. Les aptitudes évaluées durant cette étape concernent à la fois les caractéristiques des mères, les performances des produits de la naissance au sevrage, et les performances bouchères des produits mâles (Frebling et al., 1980 ; Gillard et al., 1986).

\section{Evaluation de races paternelles de croisement terminal (étape III.3 - tableau 7)}

Les femelles $G_{2}$ procréées lors de la phase II et non utilisées pour les étapes précédentes (III.1 et III.2) ou pour diverses manipulations zootechniques ont servi de

\section{TABleau 7}

Plans d'accouplements de la "phase III"

pour la comparaison de races paternelles pour le croisement terminal

Mating plans of "phase III" for comparison between sire breeds for terminal crossing

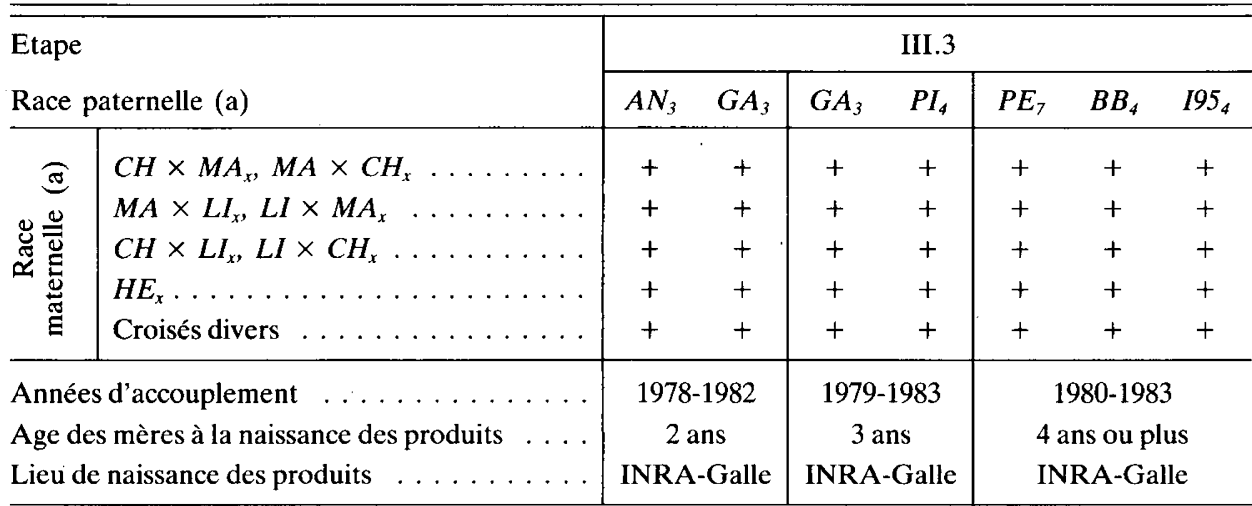

(a) $C H=$ Charolaise,$L I=$ Limousine,$M A=$ Maine-Anjou, $H E=$ Hereford,$B B=$ Blanc Bleu Belge, $P E=$ Pie-Rouge de l'Est, AN = Angus, GA = Gasconne, $P I=$ Piémontaise, $195=$ "INRA95». L'indice figurant avec le sigle de chaque race indique le nombre de pères ou mères utilisés $(x=10$ à 15 génisses provenant des étapes II.2 et III.3 et d'autorenouvellement, nées de 1977-1981). Les pères $A N$ et $G A$ sont les mêmes que ceux de l'étape III.2. Les pères $P E$ sont différents de ceux de l'étape II.3.

$\mathrm{CH}=$ Charolais, $\mathrm{LI}=$ Limousin, $\mathrm{MA}=$ Maine-Anjou, $\mathrm{HE}=$ Hereford,$\quad \mathrm{BB}=$ Blue White Belgium, $\mathrm{PE}=$ French Simmental, AN = Angus, GA = Gascon, PI = Piemontese, I95 = «INRA $95 \%$. The sign following the breed symbol specifies the number of sires or dams used $(\mathrm{x}=10$ to 15 heifers from steps 11.2 and II.3 and from self replacement, born between 1977 and 1981). The AN and GA sires are the same as those of step III.2. The PE sires are different from those of steps II.3. 
support maternel à des comparaisons de races paternelles de croisement terminal selon l'âge des mères. Pour chaque âge des mères, le choix des races paternelles a été guidé par leur taille et leur diversité de types biologiques : race à viande britannique (Angus), race rustique française (Gasconne), et race mixte cularde italienne (Piémontaise) pour les jeunes mères ; race mixte française (Pie-Rouge de l'Est), race mixte cularde belge (Blanc Bleu Belge) et lignée mâle cularde spécialisée pour le croisement terminal ( INRA $95 »-$ MéNISSIER, 1982c) pour les mères les plus âgées. Les taureaux Angus et Gascons étaient les mêmes que ceux de l'étape précédente (III.2). Pour les autres races paternelles, la plupart des pères faisaient partie de ceux utilisés dans l'essai français d'évaluation des races paternelles de croisement terminal sur vaches frisonnes (MénIsSIER et al., 1982a). Les conditions de naissance et les aptitudes bouchères des produits ainsi engendrés ont été évaluées.

\section{Conduite d'élevage et contrôles des performances}

Cette expérimentation s'est déroulée dans différentes unités expérimentales selon les étapes concernées (tableaux 2, 4, 5, 6 et 7) et les aptitudes évaluées :

- «INRA-Galle » (région Centre) et «INRA-Vieux Pin » (région Normande) pour l'élevage et la reproduction des femelles ainsi que pour certaines phases d'engraissement d'une partie de leurs produits;

— «INRA-La Minière » (région Ile-de-France) pour l'engraissement des produits mâles et des vaches de réforme ;

- «INRA-Jouy-en-Josas" (région Ile-de-France) ou «INRA-Theix » (région Auvergne) pour les abattages contrôlés.

La conduite des animaux et les modalités de contrôle seront décrites ultérieurement dans les publications relatives à l'analyse des différentes phases. Seules les grandes lignes des systèmes d'élevage seront précisées ici, sans mentionner les adaptations spécifiques à certaines années ou étapes.

\section{A. Troupeau de femelles en reproduction - "INRA-Galle" \\ (femelles : $\mathrm{G}_{0}, \mathrm{G}_{1}$ et $\mathrm{G}_{2}$; phases : I.1, I.2, II, III.1 et III.3)}

En raison de la structure du domaine expérimental, les vaches ont été conduites en stabulation libre permanente à l'exception de la période après vêlage (environ 10 à 45 jours) où elles étaient sur des parcours pour limiter les risques épidémiologiques liés a une forte concentration de jeunes veaux contemporains. Elles ont été allotées par groupe de 12 , indépendamment de leur type génétique, selon leur stade de gestation ou d'allaitement.

Pour la période hivernale (novembre à mi-avril), l'alimentation était à base d'ensilage d'herbe ou de maïs en quantité limitée et de foin ou de paille offerts à volonté, complémentés selon les besoins recommandés à l'époque (SENTEX \& Leroy, 1952, puis Petit \& Gueguen, 1978). Pour la période estivale (mi-avril à octobre), les vaches ont reçu des fourrages verts distribués à l'auge et de nature différente selon la saison. Les quantités ingérées n'ont pas été contrôlées individuellement ou par lot. 
La mise à la reproduction a eu lieu à dates fixes (mi-avril à début juillet) quels que soient le stade d'allaitement et l'âge des femelles de plus d'un an. La reproduction s'est faite par insémination artificielle sur oestrus naturels sauf pour les génisses $G_{0}$ impubères à 15 mois, les vaches $G_{0}$ en anoestrus de lactation 2,5 mois après vêlage et les génisses $G_{2}$ (phase III) pour lesquelles la mise à la reproduction a eu lieu après induction artificielle des oestrus. Pour chaque femelle, le choix du taureau a été fait au hasard parmi ceux prévus par le dispositif expérimental avec changement systématique de race d'une année sur l'autre pour les étapes expérimentales où celà était possible.

Les veaux, allaités par leurs mères, ont disposé d'une complémentation limitée systématique dès l'âge de 1,5 mois et ont été sevrés à l'âge constant de 6 mois. Après sevrage, les génisses ont d'abord subi un contrôle individuel de croissance identique à celui des mâles entiers de l'âge de 8 à 15 mois, puis ont été élevées séparément des vaches jusqu'à leur mise à la reproduction. En dehors de causes accidentelles ou pathologiques, les vaches n'ont été réformées en cours d'expérience que si elles étaient non gestantes pendant deux années consécutives. Ces vaches réformées ont été regroupées chaque automne pour être engraissées et contrôlées ( INRA-La Minière ", « INRA - Jouy-en-Josas »).

Ce système d'élevage a permis de contrôler la croissance, la morphologie, la précocité sexuelle, la fertilité, la production laitière, la capacité d'ingestion et la valeur de réforme des femelles reproductrices ainsi que les conditions de naissance, la viabilité et la croissance de leurs veaux jusqu'au sevrage.

\section{B. Troupeau de femelles en reproduction "INRA-Vieux Pin"}

(femelles : $G_{0}$ et $G_{2}$, phases : I.3 et III.2)

Comme dans les élevages à viande de la région Ouest de la France à vocation herbagère, ce troupeau a été conduit au pâturage sans complémentation alimentaire de la mi-avril à la fin octobre et en stabulation libre durant la période hivernale avec une alimentation à base d'ensilage de maïs ou d'herbe et de paille ou de foin distribués en quantité limitée et complémentés selon les besoins des animaux. Les veaux ont été sevrés à âge constant ( 7 mois) sans avoir reçu de complémentation.

La reproduction s'est faite à dates fixes par insémination artificielle sur oestrus naturels de la mi-avril à début juillet, suivi d'une période d'environ un mois de « repasse " avec un taureau en monte naturelle. Pour le premier vêlage à deux ans, les génisses de races pures nées dans ce troupeau (issues des mères $G_{0}$, phase I.3) ont été inséminées sur oestrus induits puis sur retours naturels en oestrus comme leurs contemporaines $G_{3}$ croisées trois races (issues de mères $G_{1}$, phase II.2) transférées dans ce troupeau après fécondation (phase III.3).

Les réformes ont été réalisées selon le même principe que précédemment, avec cependant un taux d'élimination plus important après le premier vêlage pour limiter l'effectif de ce troupeau.

Les contrôles ont été simplifiés et moins fréquents pour ces vaches. 


\section{Engraissement et abattage des mâles $\left(\mathrm{G}_{1}, \mathrm{G}_{2}\right.$ et $\mathrm{G}_{3}$; phases : I, II et III)}

Pour évaluer les aptitudes bouchères des mâles après sevrage, deux systèmes de production (taurillons ou bœufs) ont été utilisés selon les phases expérimentales. Les taurillons ont été engraissés et contrôlés individuellement ( INRA-La Minière ») avec un régime alimentaire à base d'aliments déshydratés (luzerne, pulpe de betteraves) distribués à volonté. Ils ont été abattus («INRA-Theix ») à âge constant soit en totalité vers 15 mois soit vers 15 ou 18 mois, selon les phases expérimentales concernées. Les contrôles ont essentiellement porté sur la vitesse de croissance, la consommation alimentaire, la morphologie et la composition des carcasses et les qualités de la viande. L'engraissement des bœufs s'est pratiqué selon un système classique de production de viande à l'herbe ( INRA-Vieux Pin»), avec une finition à l'auge de 2,5 mois environ ( INRA-La Minière ») et un abattage vers l'âge de 30 mois («INRA - Jouy-enJosas »). Contrairement aux taurillons, leur consommation alimentaire n'a pas été contrôlée individuellement.

\section{Conclusion}

En précisant le contexte, le dispositif et l'organisation de l'expérimentation, nous avons montré les possibilités et limites d'interprétation des informations fournies par cette évaluation des races à viande françaises. Du fait de sa durée de réalisation pour acquérir les résultats, il existe inévitablement un certain déphasage entre l'objectif initial fixé en fonction du contexte de l'époque et les préoccupations actuelles concernant l'exploitation du matériel génétique disponible pour la production de viande bovine. Cependant, l'originalité et l'intérêt des résultats attendus demeurent aussi bien pour l'évaluation analytique des différences d'aptitudes entre nos races à viande qui sont mal connues en race pure ou inconnues en croisement entre elles, que pour la connaissance de leurs effets génétiques. D'ailleurs, de nombreuses analyses partielles effectuées en cours de réalisation de cette expérimentation ont déjà contribué à la connaissance de la variabilité entre races et types biologiques bovins. Elles ont notamment permis une meilleure compréhension des relations entre le potentiel de croissance et les qualités maternelles. La connaissance de ces relations reste d'ailleurs aujourd'hui une des préoccupations dominantes de l'amélioration génétique des bovins à viande pour optimiser leur exploitation et déterminer leurs objectifs de sélection.

Reçu le 20 mai 1987. Accepté le 19 octobre 1987.

\section{Remerciements}

Nous remercions l'ensemble des organismes administratifs, techniques et professionnels dont les aides techniques et financières ont facilité la mise en cuvre de cette expérimentation. Nous sommes également redevables aux nombreux stagiaires qui, grâce à leur participation, ont permis de réaliser de multiples observations originales à partir de ce matériel génétique et ont contribué aux différentes analyses partielles. Que l'ensemble des chercheurs et techniciens de l'équipe d'amélioration génétique des bovins à viande à l'INRA ainsi que ceux des autres départements de recherches impliqués de près ou de loin dans cette étude durant tout son déroulement, acceptent toute notre gratitude. 


\section{Références bibliographiques}

BÉRANGer C., Ménissier F., 1981. Les races bovines allaitantes : rapport de la commission des recherches bovines de l'INRA. Bull. Tech. C.R.Z.V.-Theix, INRA, 43, 5-14.

BiBÉ B., Foulley J.L., 1976. Effets d'hétérosis chez les bovins à viande : résultats bibliographique. In : L'hétérosis : aspects théoriques et expérimentaux, Bull. Tech. Dép. Génét. Anim., INRA, 24, 87-110.

Bibé B., Frebling J., Ménissier F., 1973. Schéma d'utilisation des races rustiques en croisement avec des races à viande. In : $24^{e}$ Réunion annuelle de la Fédération Européenne de Zootechnie, Vienne, 23-27 septembre 1973, 30 p., Ann. Génét. Sél. Anim., 6, 144 (abstr.).

Bibé B., Frebling J., Ménissier F., Vissac B., 1976. Utilisation des races rustiques en croisement avec des races à viande : exemple de la race Gasconne. Ann. Génét. Sél. Anim., 8, 233-264.

Brinks B., Olson J., Caroll E.J., 1973. Calving difficulty and its association with subsequent productivity in Herefords. J. Anim. Sci., 36, 11-17.

CARTER A.H., 1975. Evaluation of cattle breeds for beef production in New-Zealand. A review. Livest. Prod. Sci., 2, 237-340.

Cartwright T.C., 1970. Selection criteria for beef cattle for the future. J. Anim. Sci., 30, 706-711.

CARTWRIGHT T.C., 1976. Use modelling cattle breeding schemes : optimization of breeding schemes and optimization of research ressources. In : Mc Gloughuin P. (ed.), Optimization of cattle breeding schemes, 153-162, Commission of the European Communities, EUR 5490e, Luxembourg.

CARTwright T.C., 1982a. Objectives in beef cattle improvement. In : Barton R.A., SMith W.C. (ed.), Proceedings of the world congress on sheep and beef cattle breeding, Canterbury, November 10-13, 1980, vol. 3, 19-27, The Dunmore Press Limited, Palmerston North.

Cartwright T.C., 1982 b. Application of systems analysis to the genetics of beef cattle production systems. In: $2^{\text {nd }}$ world congress on genetics applied to livestock production, Madrid, October 4-8, 1982, vol. 5, 504-513, Garsi, Madrid.

Cartwright T.C., Fitzhugh H.A., 1974. Efficient breeding systems for commercial beef production. In : $1^{\text {st }}$ world congress on genetics applied to livestock production, Madrid, October 1974, vol. 1, 643-653, Garsi, Madrid.

Casu S., Boyazoglu J.G., BibÉ B., Vissac B., 1975. Systèmes d'amélioration génétique de la production de viande bovine dans les pays méditerranéens : les recherches sardes. Bull. Tech. Dép. Génét. Anim. INRA, 22, 54 p.

Colleau J.J., Ménissier F., Monteiro L.S., Vissac B., 1973. Tendances actuelles de l'expérimentation en matière de sélection bovine. Ann. Génét. Sél. Anim., 5, 147-153.

Cundiff L.V., 1970. Experimental results on crossbreeding cattle for beef production. J. Anim. Sci., 30, 694-705.

CUNDIFF L.V., 1984a. Output and impact differences among divers breeds of cattle. In : HofMEYER J.H., MeYer E.H.H. (ed.), Proceedings of the $2^{\text {ni }}$ world congress on sheep and beef cattle breeding, Pretoria, April 16-19, 1984, 576-588, South African Stud Book and Livestock Improvement Association, Bloemfontein.

CunDiFF L.V., 1984b. Genetic variation and covariation between and within breeds for beef production. In: Proceedings of the 4th conference of Austr. Assoc. Anim. Breeding and Genetics, Adélaïde, June 4-6, 1984, 66-69, Australian Association of Animal Breeding and Genetics, Adélaïde.

Cundiff L.V., Gregory K.M., Косh R.E., Dickerson G.E., 1986. Genetic diversity among cattle breeds and its use to increase beef production efficiency in a temperate environment. In $: 3^{\text {rd }}$ world congress on genetics applied to livestock production, Lincoln, July 16-22, 1986, Agricultural Communications, vol. 9, 271-282, University of Nebraska, Lincoln.

Dickerson G.E., 1969. Experimental approaches in utilizing breed ressources. Anim. Breed. Abstr., 37, 191-202.

DicKerson G.E., 1970. Efficiency of animal production : modeling the biological components. $J$. Anim. Sci., 30, 849-859.

Dickerson G.E., 1973. Inbreeding and heterosis in animals. In : Proceedings of animal breeding and genetics, symposium in honor of J.L. LuSH, Blaksburg, 54-77, American society of animal science and American dairy science association, Champaign. 
Dillard E.U., Rodriguez O., Robinson O.W., 1980. Estimation of additive et non additive direct and maternal genetics effects from crossbreeding beef cattle. J. Anim. Sci., 50, 653-663.

Eisen E.J., Horstgen-Schwark G., Saxton A.M., Bandy T.R., 1983. Genetic interpretation and analysis of diallel crosses with animals. Theor. Appl. Genet., 65, 17-23.

Foulley J.L., LeForT G., 1978. Méthode d'estimation des effets directs et maternels en sélection animale. Ann. Génét. Sél. Anim., 10, 475-496.

Frebling J., Poujardieu B., Vissac B., Beranger C., Teissier J.H., 1967. Stations de sélection bovine. Compte rendu technique $\mathrm{n}^{\circ} 2$ : Etude du croisement sur la race d'Aubrac. Bull. Tech. Inf. Ing. Serv. Agric., 225, 895-906.

Frebling J., Poujardieu B., Vissac B., Azan M., Gaillard J., Rondeau M., Hennequin M., 1970. Stations de sélection bovine. Compte rendu technique $n^{\circ} 6$ : comparaison des races Charolaise et Blonde d'Aquitaine en croisement de première génération sur la race d'Aubrac. Bull. Tech. Inf. Ing. Serv. Agric., 253, 635-642.

Frebling J., Gillard P., Laissus R., Leconte D., Ménissier F., 1980. Production de bœufs de 30 mois de différentes races à viande : bilan de la $1^{\text {re }}$ série (1977-1979). Bull. Tech. C.R.Z.V. Theix, INRA, 41, 15-21.

Frebling J., Bonaïti B., Bibé B., Gillard P., Ménissier F., Renand G., 1982. Comparisons of fattening and slaughter performance between Charolais, Limousin, Maine-Anjou and Hereford breeds according to various production types. In : $2^{\text {nd }}$ world congress on genetics applied to livestock production, Madrid, October 4-8, 1982, vol. 8, 334-339, Editorial Garsi, Madrid.

Geay Y., 1986. La production de viande de taurillons. In : Micol D. (ed.), Production de viande bovine, 151-168, INRA, Paris.

Gillard P., Leconte D., Chambeyron J.J., Frebling J., 1986. Production de bœufs de différents types génétiques finis à l'auge à 30 mois (bilan de 7 années). Bull. Tech. C.R.Z.V. Theix, INRA, 65, 31-39.

Gregory K.E., Cundiff L.V., 1980. Crossbreeding in beef cattle : evaluation of systems. J. Anim. Sci., 51, 1224-1242.

Gregory K.E., Cundiff L.V., Koch R.M., CASTer D.B., Sмith G.M., 1978. Heterosis and breed maternal and transmitted effects in beef cattle. 1. Preweaning traits. J. Anim. Sci., 47, 10311041.

HAYS W.G., BRINKS J.S., 1980. Relationship of weight and height to beef cow productivity. $J$. Anim. Sci., 50, 793-799.

HoHENBOKEN W.D., BrINKS J.S., 1971. Relationships between direct and maternal effects on growth in Herefords. 3. Covariance of paternal half-brother and sister performance. J. Anim. Sci., 32, 35-42.

Joandet G.E., 1977. Crossbreeding research in South America. In : Mason I.L., Pabst W. (ed.), Crossbreeding experiments and strategy of beef utilization to increase beef production, 155-170, Commission of the European Communities. EUR 5492e, Luxembourg.

Joandet G.E., Cartwright T.C., 1975. Modeling beef cattle production systems. J. Anim. Sci., 41, 1238-1246.

Koch R.M., Dickerson G.E., Cundiff L.V., Gregory K.E., 1985. Heterosis retained in advanced generations of crosses among Angus and Hereford cattle. J. Anim. Sci., 60, 1117-1132.

LiboRIusSeN T., 1982. Comparison of paternal strains used in crossing and their interest for increasing production in dairy herds. In: $2^{\text {nd }}$ world congress on genetics applied to livestock Production, Madrid, October 4-8, 1982, vol. 5, 469-481, Editorial Garsi, Madrid.

LoNG C.R., 1980. Crossbreeding for beef production: experimental results. J. Anim. Sci., 51, 1197-1223.

MANGUS W.L., Brinks J.S., 1971. Relationships between direct and maternal effects on growth in Herefords : 1. Environmental factors during preweaning growth. J. Anim. Sci., 32, 17-25.

MASON I.L., PABST W., 1977. Crossbreeding experiments and strategy of beef utilization to increase beef production. 490 p., Commission of the European Communities, EUR 5492e, Luxembourg.

MÉNISSIER F., 1975a. Genetic aspects related to use of beef breeds. In : TAYLER J.C. (ed.), The early calving of heifers and its impact on beef production, 81-122, Commission of the European Communities, EUR 5545e, Brussels.

MÉNISSIER F., 1975b. Calving ability in French beef breeds : analysis of components and breeding improvement. In : MÉnissier F., Vissac B., Frebling J. (ed.), Optimum breeding plans for beef cattle. Bull. Tech. Dép. Génét. Anim., INRA, 21, 58-102. 
MÉNISSIER F., 1982a. Beef cattle genetics and breeding : general state, evolution and prospect for temperate areas. In : $2^{\text {nd }}$ world congress on genetics applied to livestock production, Madrid, October 4-8, 1982, vol. 5, 449-468, Editorial Garsi, Madrid.

MÉNISSIER F., 1982b. Present state of knowledge about the genetic determination of muscular hypertrophy or double-muscled trait in cattle. In : KING J.W.B., MÉnissier F. (ed.), Muscle hypertrophy of genetic origin and its use to improve beef production, 387-428, Martinus Nijhoff, The Hague.

MÉnissier F., 1982c. Advantages of using double muscled sires in crossbreeding and the selection of a specialized double-muscled sire line in France. In : King J.W.B., MÉnISsier F. (ed.), Muscle hypertrophy of genetic origin and its use to improve beef production, 480-536, Martinus Nijhoff, The Hague.

Ménissier F., Foulley J.L., 1979. Present situation of calving problems in the E.E.C. : incidence of calving difficulties and early calf mortality in beef herds. In : HofFman B., Mason I.L., Schmir J. (ed.), Calving problems and early viability of the calf, 30-85, Martinus Nijhoff, The Hague.

Ménissier F., Bibé B., Perreau B., 1974. Possibilités d'amélioration des conditions de vêlage par sélection. 2. Aptitude au vêlage de trois races à viande françaises. Ann. Génét. Sél. Anim., 6, 69-90.

Ménissier F., Vissac B., Frebling J., 1975. Optimum breeding plans. In : Ménissier F., VisSac B., Frebling J., (ed.), Optimum breeding plans for beef cattle. Bull. Tech. Dép. Génét. Anim., INRA, 21, 4-56.

Ménissier F., Sapa J., Foulley J.L., Frebling J., Bonaïti B., 1982a. Comparison of different sire breeds crossed with Friesian cows : preliminary results. In : MoRE O'FERrall G.J. (ed.), Beef production from different dairy breeds and dairy beef crosses, 94-136, Martinus Nijhoff, The Hague.

Ménissier F., Frebling J., Perreau B., Gillard P., Pourtier D., 1982b. Comparison of sire breeds (Limousin, Aberdeen-Angus, Jersey) used in crossbreeding for early calving in French beef heifers. In : $2^{\text {nd }}$ world congress on genetics applied to livestock production, Madrid, October 4-8, 1982, vol. 8, 340-345. Editorial Garsi, Madrid.

More O'Ferrall G.J. (ed.), 1982. Beef production from different dairy breeds and dairy beef crosses. 395 p., Martinus Nijhoff, The Hague.

PETrT M., 1975. Early calving in suckling herds. In: TAYLER J.C. (ed.), The early calving of heifers and its impact on beef production, 157-176, Commission of the European Communities, EUR 5545e, Brussels.

Petit M., Gueguen L., 1978. Vaches nourrices. In : JARrige R. (ed.), Alimentation des ruminants, 377-402, INRA, Paris.

Petit M., Trillat G., Dubroeuce H., Sauvaire A., 1973. Possibilités de faire vêler à deux ans les génisses destinées à la boucherie. Bull. Tech. C.R.Z.V., Theix, INRA, 13, 5-18.

Rastogi R., Chevalet C., 1973. On methods of estimation of maternal heterosis and recombination effects from a specific three breed crossing system. Ann. Génét. Sél. Anim., 5, 495-498.

SenteX J., Leroy A.M., 1952. L'alimentation des animaux à la portée de tous: équivalents fourragers. 80 p., Comité fédératif national de contrôle laitier, Paris.

TAYLER J.C. (ed.), 1975. The early calving of heifers and its impact on beef production. 295 p., Commission of the European Communities. EUR 5545e, Brussels.

TAYLER J.C., 1976. Beef production in the E.E.C. and the coordination of research by the Commission of the Communities. Livest. Prod. Sci., 3, 305-318.

Vissac B., 1976a. Possibilités et limites d'utilisation des races à fort potentiel de croissance musculaire pour améliorer la production de viande bovine dans le monde. World Rev. Anim. Prod., 19, 1-7.

VISSAC B., 1976b. Optimizing cattle breeding in E.E.C. : some ideas and schemes from the French situation. Tierzüchter, 28, 486-488, 530-534.

Vissac B., Bonhomme D., Frebling J., 1971. L'utilisation des races à viande françaises en croisement de première génération pour la production de veaux de boucherie : bilan de dix années de recherche (1960-1970). Bull. Tech. Dép. Génét. Anim., INRA, 12, 27 p.

Wilton J.N., Morris C.A., Jenson E.A., Leigh A.O., Pfeiffer W.C., 1974. $\Lambda$ linear programming model for beef cattle production. Can. J. Anim. Sci., 54, 693-708. 